\title{
Notes on the Floquet-Lyapunov Theory of Linear Differential Systems
}

\author{
Kai Zenger \\ Department of Electrical Engineering and Automation, Aalto University, Finland, kai.zengereaalto.fi
}

\begin{abstract}
This short remark describes some of the key points of the Floquet-Lyapunov theory of linear periodic differential systems. The basic transformation is presented, and its properties from the point of view of the source and target matrices and stability are discussed. The proposed methodology can be used in analysis, control design and simulation of systems with time-periodic characteristics. A method for designing a stabilizing state feedback control law is proposed. The methodology can be used in analysis and controller design for example in processes involving rotating machines and engines.
\end{abstract}

Keywords: time periodic systems, Floquet-Lyapunov transformation, monodromy matrix, stability

\section{Introduction}

The analysis and controller design of time-varying systems is difficult even in the case that the system or its model can be approximated to be linear. Such systems are for example rotating machines with changing speed or chemical reactors with changing flow rates and liquid volumes. Mathematically the models of such systems contain time-varying parameters. That is the case with the inputoutput mappings, which for continuous time differential models means differential equations or state-space representations.

The fundamental reason for the difficulties in analysis is that the solution of the time-varying differential system can usually not be expressed by elementary functions (Rugh, 1993). In system-theoretic terms this fact can be noted by the fact that the state transition matrix cannot be solved analytically. This function (or the solution of the system equation) exists but unlike in the time invariant case it cannot be expressed by elementary functions.

Input-output systems are usually modelled by statespace representations. Generally, the matrices involved (system matrix, input and output matrices) contain values that change with time. It is well-known that with a suitable transformation of the state variable (change of coordinates) exists such that the system matrix in the new coordinates becomes constant (Harris and Miles, 1980). Generally, the time-varying transformation matrix between the state variables is then complex, which is unfortunate when considering the potential use of the transformation for controller design. Anyway the original and the new state-space representation are kinematically similar under the state transformation $x(t)=P(t) s(t)$, where $P(\cdot)$ is the transformation matrix (Harris and Miles, 1980). Further, if $P(\cdot)$ is a Lyapunov transformation the stability properties of the original and new state-space representation are known to be the same. The matrix is a Lyapunov transformation if it is invertible and both the norms of the matrix and its inverse are bounded for all time instants (Lyapunov, 1966). A fundamental problem is the fact that a suitable transformation is hard to find.

In short: Given a system matrix $A(\cdot)$ and choosing another matrix $E(\cdot)$ with the same dimension there exists a time-varying similarity transformation $P(\cdot)$ that changes $A(\cdot)$ to $E(\cdot)$. However, $P(\cdot)$ is not necessarily a Lyapunov transformation. Choosing $P(\cdot)$ in advance to be a Lyapunov transformation gives $E(\cdot)$ with the same stability properties as $A(\cdot)$. But $E(\cdot)$ is then determined by the given transformation and cannot be chosen freely,

The situation is slightly less complicated, if the original system matrix is periodic. Then it is known (Wiberg, 1971; Harris and Miles, 1980; Zenger and Ylinen, 2008) that a Lyapunov function exists which changes the original system matrix into a constant matrix. However, this function is not nesearily real. The purpose of this paper is to study real Lyapunov transformations, using homogenous systems first. Later, a synthesis approach is derived for designing stabilizing control algorithms for input-output systems. The general theory is called Floquet-Lyapunov theory due to G. Floquet who invented it and A. M. Lyapunov who investigated the stability of periodic systems. The basic ideas are presented first, leading to the very important concept of the monodromy matrix. The characteristics of the state transformation and the target (constant) system matrix are studied in detail, and it is shown how a given periodic Lyapunov function and constant system matrix $E$ can be used to characterize the original timevarying system matrix and the solution of the related differential equation. The theory leads in a natural way to methods, where equations needed in synthesis problems, i.e. controller design problems, can be formulated.

\section{Linear periodic differential systems}

Consider the autonomous differential system

$$
\dot{x}(t)=A(t) x(t), \quad x\left(t_{0}\right)=x_{0}
$$


where $x(\cdot) \in\left(\Re^{n}\right)^{\Re}$ and for all $t$ it holds $A(t+T)=A(t)$ for some positive value $T$. The system matrix $A(\cdot) \in$ $\left(\Re^{n \times n}\right)^{\Re}$ is then $T$-periodic. (To be precise, $T$ must be the smallest positive number that fulfils $A(t+T)=A(t)$.) It is assumed that the function $A(\cdot)$ is continuously differentiable, which guarantees that the solution to (1) is continuously differentiable as well. Actually, the assumption could be relaxed to allow piecewise continuous function $A(\cdot)$, meaning that the solution is still unique and continuous.

Let

$$
x(t)=P(t) s(t)
$$

where $P(\cdot) \in\left(\Re^{n \times n}\right)^{\Re}$ is a continuously differentiable and invertible function for all $t$. Assume also that both $P$ and its inverse are bounded for all $t$. Easy calculations give (see (Bittanti, 2008; Zenger and Ylinen, 2008))

$$
\begin{gathered}
\dot{s}(t)=E(t) s(t) \\
\dot{P}(t)=A(t) P(t)-P(t) E(t) \\
P(t)=\Phi_{A}\left(t, t_{0}\right) P\left(t_{0}\right) \Phi_{E}\left(t, t_{0}\right)^{-1}=\Phi_{A}(t, 0) P(0) \Phi_{E}(t, 0)^{-1} \\
\Phi_{A}(t, \tau)=P(t) \Phi_{E}(t, \tau) P(\tau)^{-1}
\end{gathered}
$$

where $\Phi_{A}(\cdot, \cdot)$ and $\Phi_{E}(\cdot, \cdot)$ are the state transition matrices related to $A(\cdot)$ (source matrix) and $E(\cdot)$ (target matrix), respectively. Note that the matrix $E(\cdot)$ is determined by $A(\cdot)$ and the transformation $P(\cdot)$. But it is also possible to choose the target system matrix $E(\cdot)$ freely (same dimension as with $A(\cdot)$ ), which then determines $P(\cdot)$.

The following question now arises: when is the matrix $P$ T-periodic? To answer, note that

$$
\begin{aligned}
& P(t+T)=\Phi_{A}\left(t+T, t_{0}\right) P\left(t_{0}\right) \Phi_{E}\left(t+T, t_{0}\right)^{-1} \\
& \quad=\Phi_{A}(t+T, t) \Phi_{A}\left(t, t_{0}\right) P\left(t_{0}\right)\left[\Phi_{E}(t+T, t) \Phi_{E}\left(t, t_{0}\right)\right]^{-1} \\
& \quad=\Phi_{A}(t+T, t) \underbrace{\Phi_{A}\left(t, t_{0}\right) P\left(t_{0}\right) \Phi_{E}\left(t, t_{0}\right)^{-1}}_{P(t)} \Phi_{E}(t+T, t)^{-1}
\end{aligned}
$$

By introducing the monodromy matrix

$$
\Psi_{A}(t)=\Phi_{A}(t+T, t)
$$

we obtain

$$
P(t+T)=\Psi_{A}(t) P(t) \Psi_{E}(t)^{-1}
$$

The condition that $P(\cdot)$ is $T$-periodic is that for all $t$ it holds $P(t+T)=P(t)$. Then

$$
\Psi_{A}(t) P(t) \Psi_{E}(t)^{-1}=P(t) \Rightarrow \Psi_{A}(t)=P(t) \Psi_{E}(t) P(t)^{-1}
$$

or

$$
\Phi_{A}(t+T, t)=P(t) \Phi_{E}(t+T, t) P(t)^{-1}
$$

which must hold for all $t$. The equation (10) is the condition that must be fulfilled for the transformation matrix $P(\cdot)$ to be $T$-periodic. Further, we can see from (9) that the monodromy matrices $\Psi_{A}(\cdot)$ and $\Psi_{E}(\cdot)$ are similar at all time instants. That implies that their eigenvalues are the same at each time instant. Note that in the derivations of equations (9) and (10) no assumptions on the periodicity of the matrices $A(\cdot)$ and $E(\cdot)$ have been made. The result is then general.

But assuming that $A(\cdot)$ is $T$-periodic we can go further by noticing that

$$
\begin{aligned}
\Psi_{A}\left(t_{1}\right) & =\Phi_{A}\left(t_{1}+T, t_{1}\right)=\Phi_{A}\left(t_{1}+T, t_{2}\right) \Phi_{A}\left(t_{2}, t_{1}\right) \\
& =\Phi_{A}\left(t_{1}+T, t_{2}+t\right) \Phi_{A}\left(t_{2}+T, t_{2}\right) \Phi_{A}\left(t_{2}, t_{1}\right) \\
& =\Phi_{A}\left(t_{1}, t_{2}\right) \underbrace{\Phi_{A}\left(t_{2}+T, t_{2}\right)}_{\Psi_{A}\left(t_{2}\right)} \Phi_{A}\left(t_{1}, t_{2}\right)^{-1}
\end{aligned}
$$

where the important result that for the state transition matrix corresponding a $T$-periodic matrix $A(\cdot)$ it holds $\Phi_{A}\left(t_{1}+T, t_{2}+T\right)=\Phi_{A}\left(t_{1}, t_{2}\right)$ for all $t_{1}, t_{2}$ has been used. (See the Remark below.) It follows that at all time instants $t_{1}$ and $t_{2}$ the monodromy matrices $\Psi_{A}\left(t_{1}\right)$ and $\Psi_{A}\left(t_{2}\right)$ are similar. Hence, their eigenvalues are the same for all $t$ meaning that the eigenvalues are constant.

So, if $P(\cdot)$ and $A(\cdot)$ are $T$-periodic, the eigenvalues of $\Psi_{A}(\cdot)$ and $\Psi_{E}(\cdot)$ are equal and constant.

Remark 1: The fact that the state transition matrix a $T$-periodic system matrix $A(t)$ fulfils $\Phi_{A}(t+T, \tau+T)=$ $\Phi_{A}(t, \tau)$ for all $t$ and $\tau$ follows by noticing that

$$
\begin{aligned}
& \frac{\partial \Phi_{A}(t+T, \tau+T)}{\partial(t+T)}=A(t+T) \Phi_{A}(t+T, \tau+T) \\
& =A(t) \Phi_{A}(t+T, \tau+T) \\
& \Phi_{A}(t+T, \tau+T)_{\mid t=\tau}=I
\end{aligned}
$$

and on the other hand

$$
\begin{aligned}
& \frac{\partial \Phi_{A}(t, \tau)}{\partial t}=A(t) \Phi_{A}(t, \tau) \\
& \Phi_{A}(t, \tau) \\
& \mid t=\tau=I
\end{aligned}
$$

Both functions $\Phi_{A}(t+T, \tau+T), \Phi_{A}(t, \tau)$ satisfy the same differential equation and have one common point $(=\mathrm{I})$. Hence, the functions are equal.

Remark 2: Note that the condition for $P(\cdot)$ given in (5) holds generally in the sense that for all $t, t_{1}$

$$
\begin{aligned}
P(t) & =\Phi_{A}\left(t, t_{1}\right) \Phi_{A}\left(t_{1}, t_{0}\right) P\left(t_{0}\right)\left[\Phi_{E}\left(t, t_{1}\right) \Phi_{E}\left(t_{1}, t_{0}\right)\right]^{-1} \\
& =\Phi_{A}\left(t, t_{1}\right) \underbrace{\Phi_{A}\left(t_{1}, t_{0}\right) P\left(t_{0}\right) \Phi_{E}\left(t_{1}, t_{0}\right)^{-1}}_{P\left(t_{1}\right)} \Phi_{E}\left(t, t_{1}\right)^{-1} \\
& =\Phi_{A}\left(t, t_{1}\right) P\left(t_{1}\right) \Phi_{E}\left(t, t_{1}\right)^{-1}
\end{aligned}
$$

In short: provided that $A(\cdot)$ is $T$-periodic, $E(\cdot)$ can be chosen arbitrarily as long as it is $T$-periodic also. Then the condition that for all $t(10)$

$$
\Phi_{A}(t+T, t)=P(t) \Phi_{E}(t+T, t) P(t)^{-1}
$$

guarantees the periodicity of $P(\cdot)$. Moreover, if the above condition is fulfilled at some time instant $t_{1}$, it is satisfied for all $t$. 
If a constant target matrix $E$ is chosen, the periodicity of $P(\cdot)$ is guaranteed by

$$
\begin{aligned}
\Phi_{E}(t+T, t) & =e^{\int_{t}^{t+T} E d \tau}=e^{E(t+T-t)}=e^{E T} \\
& \Rightarrow e^{E T}=P(t)^{-1} \Phi_{A}(t+T, t) P(t)
\end{aligned}
$$

which must hold for all $t$ so without loss of generality it is possible to take $t=0$

$$
e^{E T}=P(0)^{-1} \Phi_{A}(T, 0) P(0)
$$

Further, let us remember the Jacobi-Liouville formula

$$
\operatorname{det} \Phi_{A}(t, \tau)=e^{\int^{t} \operatorname{tr}(A(v)) d v}
$$

and use it in (12)

$$
\begin{aligned}
\operatorname{det} \Phi_{A}(t+T, t) & =e^{\iint_{t}^{t+T} \operatorname{tr}(A(v)) d v} \\
& =\operatorname{det} P(t) \cdot \operatorname{det} \Phi_{E}(t+T, t) \cdot \underbrace{\operatorname{det} P(t)^{-1}}_{1 / \operatorname{det} P(t)} \\
& =e^{\int_{t}^{t+T} \operatorname{tr}(E(v)) d v}
\end{aligned}
$$

It follows that

$$
\int_{t}^{t+T} \operatorname{tr}(A(v)-E(v)) d v=0
$$

which must be fulfilled for all $t$. The result gives a necessary relationship between the periodic matrices $A(\cdot)$ and $E(\cdot)$. The condition is not sufficient.

It should be emphasized that although for a $T$-periodic system matrix $A(\cdot)$ it holds

$$
\Phi_{A}(t+T, \tau+T)=\Phi_{A}(t, \tau)
$$

that does not mean that the solution $x(t)$ would be periodic. In fact

$$
\begin{aligned}
x(t+T) & =\Phi_{A}\left(t+T, t_{0}\right) x_{0} \\
& =\Phi_{A}\left(t+T, t_{0}+T\right) \Phi_{A}\left(t_{0}+T, t_{0}\right) x_{0} \\
& =\Phi_{A}\left(t, t_{0}\right) \Psi_{A}\left(t_{0}\right) x_{0}
\end{aligned}
$$

It follows that if one of the eigenvalues of the monodromy matrix $\Psi_{A}\left(t_{0}\right)$ is equal to 1 , then choosing the initial condition $x_{0}$ to be the corresponding eigenvector leads to a periodic solution. Also, it is easy to see from the above equations that if a solution (state transition matrix) is known for one time period $\left[t_{0}, t_{0}+T\right]$ it can then be determined for all $t$.

\section{Stability of periodic systems}

Stability of periodic systems is the natural question to be discussed next. To that end take $E$ as a constant matrix and so

$$
e^{E T}=P(t)^{-1} \Phi_{A}(t+T, t) P(t)=P(t)^{-1} \Psi_{A}(t) P(t)
$$

or equivalently

$$
e^{E T}=P(0)^{-1} \Phi_{A}(T, 0) P(0)=P(0)^{-1} \Psi_{A}(0) P(0)
$$

The condition guarantees that $P(\cdot)$ is periodic and thus a Lyapunov transformation, which is known to preserve the stability properties between the original system matrix $A(\cdot)$ and target $E(\cdot),($ Rugh, 1993). The stability of $E$ then determines the stability of $A(\cdot)$ as well. But the eigenvalues of $\exp (E T)$ (characteristic multipliers) are the constant eigenvalues of the monodromy matrix $\Psi_{A}(t) . E$ is asymptotically stable, if and only if all its eigenvalues (characteristic exponents) are on the left half plane. But if $\lambda_{E}$ is an eigenvalue of $E$ and $\lambda$ is the corresponding eigenvalue of $\exp (E T)$ then it holds

$$
\lambda=e^{\lambda_{E} T}
$$

and it follows that the system is asymptotically stable, if and only if all eigenvalues of the monodromy matrix $\Psi_{A}(t)$ are inside the unit circle.

The same result can easily be obtained by looking at the properties of the monodromy matrix. For all real numbers $\tau$ and integer values $k$ it holds

$$
\Psi_{A}(\tau+(k+1) T)=\Psi_{A}(\tau)
$$

To see this, write

$$
\begin{aligned}
\Psi_{A}(\tau+(k+1) T)= & \Phi_{A}(\tau+(k+2) T, \tau+(k+1) T) \\
= & \Phi_{A}(\tau+(k+1) T, \tau+k T) \\
& =\ldots=\Phi_{A}(\tau+T, \tau)=\Psi_{A}(\tau)
\end{aligned}
$$

The solution of the state equation at time $\tau+(k+1) T$ becomes

$$
\begin{aligned}
x(\tau+(k+1) T)= & \Phi_{A}(\tau+(k+1) T, \tau+k T) x(\tau+k T) \\
= & \Psi_{A}(\tau+k T) x(\tau+k T)=\Psi_{A}(\tau) x(\tau+k T) \\
= & \Psi_{A}(\tau)^{2} x(\tau+(k-1) T) \\
& =\ldots=\Psi_{A}(\tau)^{k+1} x(\tau)
\end{aligned}
$$

The eigenvalues of the monodromy matrix must then clearly be less than one in magnitude, if the system is asymptotically stable.

Example: Consider the system matrix

$$
A(t)=\left[\begin{array}{cc}
1 & \cos (t) \\
0 & 0
\end{array}\right]
$$


where clearly $T=2 \pi$. The state transition matrix is

$$
\begin{aligned}
& \Phi_{A}(t, \tau) \\
& =\left[\begin{array}{cc}
e^{t-\tau} & (1 / 2) e^{t-\tau}(\cos (\tau)-\sin (\tau))+(1 / 2)(\sin (t) \\
0 & 1
\end{array}\right] \\
& \text { The monodromy matrix is } \\
& \qquad \Psi_{A}(t)=\Phi_{A}(t+T, t) \\
& =\left[\begin{array}{cc}
e^{T} & (1 / 2) e^{T}(\cos (t)-\sin (t))+f_{1}(t) \\
0 & 1
\end{array}\right] \\
& =\left[\begin{array}{cc}
e^{T} & (1 / 2) e^{T}(\cos (t)-\sin (t))+f_{2}(t) \\
0 & 1
\end{array}\right] \\
& =\left[\begin{array}{cc}
e^{T} & \left(1-e^{T}\right) f_{2}(t) \\
0 & 1
\end{array}\right]
\end{aligned}
$$$$
=\left[\begin{array}{cc}
e^{t-\tau} & (1 / 2) e^{t-\tau}(\cos (\tau)-\sin (\tau))+(1 / 2)(\sin (t)-\cos (t)) \\
0 & 1
\end{array}\right] \begin{aligned}
& \dot{x}(t)=(A(t)-B(t) L(t)) x(t)=A_{c l}(t) x(t) \\
& y(t)=(C(t)-D(t) L(t)) x(t)
\end{aligned}
$$

where

$$
\begin{gathered}
f_{1}(t)=(1 / 2)(\sin (t+T)-\cos (t+T)) \\
f_{2}(t)=(1 / 2)(\sin (t)-\cos (t))
\end{gathered}
$$

The eigenvalues are $e^{T}$ and 1 , and the system is unstable. That can also be seen from the state transition matrix directly.

\section{Control design}

The above theory provides the background information and tools for analysing and simulating time-periodic differential systems. It was shown that any system matrices $A(\cdot)$ and $E(\cdot)$ with equal dimensions are equivalent to each other through a real transformation matrix $P(\cdot)$. However, it is very difficult to determine this matrix. Even worse, it is not clear that a such matrix would be stability preserving (Lyapunov transformation) between the source and target system matrices.

For periodic systems things turn out to be simpler. For example, the target system matrix can be a constant real matrix and the transformation a periodic Lyapunov transformation. According to equation (24) or

$$
\Psi_{A}(t)=P(t) e^{E T} P^{-1}(t)
$$

it is possible to pick any real (stable or unstable) matrix $E$ and a periodic Lyapunov transformation $P(\cdot)$ to calculate the monodromy matrix of the source system. This fact can be used to generate a stabilizing controller for timeperiodic systems. To see that, consider the state-space representation of an input-output system

$$
\begin{aligned}
& \dot{x}(t)=A(t) x(t)+B(t) u(t) \quad x\left(t_{0}\right)=x_{0} \\
& y(t)=C(t) x(t)+D(t) u(t)
\end{aligned}
$$

where the matrices $A(\cdot), B(\cdot), C(\cdot)$ and $D(\cdot)$ are timeperiodic with the period $T$. Consider a time-varying state feedback control law

$$
u(t)=-L(t) x(t)
$$

where the gain matrix $L(\cdot)$ is $T$-periodic also. The closedloop system can be written as

and the system matrix $A_{c l}(\cdot)$ is periodic.

The idea is to pick an asymptotically stable constant real matrix $E$ and a Lyapunov transformation matrix $P(\cdot)$. If the controller gain is chosen such that the closed loop system matrix is kinematically similar to the matrix $E$, a stabilizing contol law has been found. The monodromy matrix of the closed loop (which does not even have to be calculated) can be represented as

$$
\psi_{A_{c l}}(t)=P(t) e^{E T} P(t)^{-1}
$$

But now according to (4)

$$
\begin{aligned}
& \dot{P}(t)=A_{c l}(t) P(t)-P(t) E \\
& \Rightarrow A_{c l}(t)=(\dot{P}(t)+P(t) E) P(t)^{-1}
\end{aligned}
$$

and, using the expression for $A_{c l}$

$$
\begin{aligned}
B(t) L(t) & =A(t)-A_{c l}(t) \\
& =A(t)-(\dot{P}(t)+P(t) E) P(t)^{-1}
\end{aligned}
$$

the condition for $L(\cdot)$ has been formed. However, it should be noticed that the number of states is usually larger that the number of inputs, meaning that the equation does not necessarily have a solution. There are two alternatives: An approximative solution using pseudoinverse can be used or the matrices $E$ and $P(\cdot)$ can be used as a parameterization for an existing solution for $L(\cdot)$. The former is a practical solution, which does not guarantee stability. The latter is not a direct algorithm for solving the controller gain, but shows the conditions (parameterization) which can be used. As such, it can be compared to the corresponding LMI conditions presented e.g. by (Bittanti, 2008).

\section{Conclusion}

A short introduction to the Floquet-Lyapunov theory of periodic linear differential systems was given in the paper. It was shown that the results are reasonably easy to derive by starting from a transformation between source and target differential systems. Although the theory is not new, see e.g. (Bittanti, 2008) it is believed to be more easy to come up with by considering the presented approach. Also, using the characteristics of the theory a way to design stabilizing control laws for periodic systems was presented. In a way, the method is used to parameterize system matrices of periodic differential systems. The results are easy to extend to periodic discrete-time systems as well.

\section{References}

Sergio Bittanti. Periodic systems : filtering and control. Springer, New York, 2008. 
C. J. Harris and J. F. Miles. Stability of Linear Systems: Some Aspects of Kinematic Similarity. Academic Press, New York, 1980.

A. M. Lyapunov. Stability of Motion. Academic Press, New York, 1966.

W. J. Rugh. Linear system theory. Prentice-Hall, Inc., Englewood Cliffs, New Jersey, 356 p., 1993. ISBN 0-13-555038-6.

D. M. Wiberg. State Space and Linear Systems. McGraw-Hill, New York, 1971.

K. Zenger and R. Ylinen. Representations with constant system matrices of linear time-periodic dynamical systems. In 17th World Congress of the International Federation of Automatic Control, Seoul, Korea, 2008. 